\title{
La expresión musical al servicio de la integración del conocimiento: Música en Educación Secundaria y currículum en red
}

\author{
Musical Expression at the Heart of Meshed Knowledge: Music and \\ Integrated Curriculum in Secondary Education
}

Macarena PÉREZ. Universitat de València.macarena_p3@yahoo.es

\begin{abstract}
Resumen: Este artículo, extraído de mi Trabajo Final del Máster en Profesor/a de Educación Secundaria, propone la organización en red de los contenidos del currículum de Secundaria correspondientes a la asignatura de Música. Se trata de un modelo muy desarrollado durante las últimas décadas por teóricos de la didáctica de las artes visuales como A. D. Efland, que consiste en la creación de retículas mediante la interconexión de conceptos. Su objetivo es potenciar la capacidad de análisis y relación de los estudiantes, así como transmitir una imagen más fiel del carácter interdisciplinar de las materias. Otros beneficios que se desprenden del currículum integrado, como se lo conoce también, son el aumento de la implicación del alumno en una dinámica de clase que se establece como dialogadora e interactiva, y el impulso de las competencias básicas. Tras un breve recorrido por las teorías del aprendizaje que desembocan en el concepto de currículum en red, analizo las ventajas de su implementación en la asignatura de música y expongo dos aplicaciones prácticas del mismo: un sistema de coordenadas que permite integrar las diferentes modalidades de praxis musical, por una parte, y una propuesta didáctica, por otra. En definitiva, este trabajo nace de la convicción de que el modo en que se enseña es tan importante como lo que se enseña, o mejor, de que el modo en que se aprende es tan importante como lo que se aprende.
\end{abstract}

Palabras clave: música, currículum, red, integración, interacción, competencias

Abstract: This paper, which is part of my master's thesis in Secondary Education, puts forward a way of structuring into a network the contents to be taught in Music at the secondary level. Such an approach has been largely developed in the teaching of visual arts by scholars like A. D. Efland and is about weaving 
reticles by interconnecting concepts. Its aim is to favour the analytical skills of the students and their ability to associate, as well as to give a more faithful image of the interdisciplinary nature of the different fields of knowledge. Furthermore, integrated curriculum, as it is known, encourages students to get involved in class dynamics based on dialogue and interactivity and therefore stimulates the acquisition of basic skills. After briefly examining the learning theories that underlie it, I discuss the advantages of implementing integrated curriculum in music teaching. I then put forward a coordinate system which allows for the integration of the different modalities of musical practice and a didactic proposal in line with that same principle. In short, this paper emerges from the conviction that the way we teach is just as important as what we teach, or rather, that the way we learn is just as important as what we learn.

Keywords: music, curriculum, network, integration, interactivity, competence

\section{Introducción}

Tan pronto como el profesor Ferran Roda nos habló en su clase de Procesos y Contextos Educativos sobre el currículum en red, me sentí identificada con el concepto de educación que le subyace. El alcance del currículum integrado se me reveló como un buen motivo para indagar en el tema. Al emprender la búsqueda bibliográfica, advertí que hay mucha literatura sobre currículum integrado en el campo de la enseñanza de las artes visuales, no así en el de la música. Me pareció entonces pertinente realizar un Trabajo Final de Máster que valorara la aplicabilidad de la retícula a la materia de Música en Secundaria.

Del actual currículum de Secundaria de la Comunidad Valenciana, se desprende que la asignatura de Música debe implicar mucho más que aspectos formales y estilísticos. En la relación de contenidos incluye aspectos sociales como los contextos en los que la música es protagonista, o los diversos usos que se hacen de ella. Asimismo, los bloques de contenidos abarcan diferentes modalidades de práctica musical - creación, interpretación, audición y teorización. Pero subrayo, las abarcan, no integran. El modo y el grado en que se integren los contenidos en la práctica quedan en manos del profesor.

El currículum de Música está formulado de manera muy generalizadora, que establece un marco de acción poco definido y otorga al docente un margen relativamente amplio de maniobra. Esto nos permite poner en práctica métodos didácticos alternativos y explorar sus resultados, adoptando la postura de profesores investigadores.

En épocas de crisis, cuando lo que se busca son pruebas de la productividad y la eficacia del sistema, se tiende a apostar por técnicas de instrucción que dividen los 
aprendizajes complejos en pequeños pasos fácilmente evaluables, asegurando un cierto éxito pedagógico y la evidencia de resultados positivos. Con ello, se modera con toda probabilidad el clima para la innovación educativa (Eisner, 1987) y se renuncia a un nivel de comprensión más profundo de la materia. Por eso insisto en que hemos de aprovechar al máximo el hecho de que el actual currículum de Música proporciona unas condiciones óptimas para la experimentación.

Antes de pasar a exponer algunas posibilidades de implementación del concepto de currículum en red en la asignatura de Música, resumiré a modo de breve marco teórico en los dos apartados que siguen su filosofía y antecedentes.

\section{El concepto de esquema de Piaget a Efland}

«El término "esquema" es apto para caracterizar el conocimiento porque la esencia del conocimiento es estructura. El conocimiento no es una cesta de hechos.»

\section{R. Anderson (Efland, 2004)}

La estructuración de los contenidos en retícula que defiende Arthur D. Efland se basa en la idea de esquema impulsada por Jean Piaget y ulteriormente desarrollada por teóricos como Richard Anderson y Barry J. Wadsworth. No se trata de un esquema arboriforme, donde las categorías se subdividen y alejan progresivamente unas de otras, sino de un esquema que elucida precisamente las relaciones mutuas entre todas ellas.

Wadsworth explica el desarrollo progresivo de los esquemas de conocimiento a través de los conceptos de asimilación y acomodación. El primero es la ampliación de los esquemas mediante la incorporación de nuevos contenidos; el segundo, la modificación estructural de esos esquemas mediante la creación de nuevas conexiones o bien para dar cabida a contenidos que no encajaban hasta entonces. La asimilación, en tanto que aumento en la cantidad de conocimiento asimilado, es un progreso cuantitativo, mientras que la acomodación, en tanto que proceso por el cual ese conocimiento se sitúa y organiza en red, supone un progreso cualitativo. En resumen, estos autores conciben el desarrollo cognitivo del individuo como el proceso por el cual sus esquemas mentales se hacen más numerosos al tiempo que más interconexos.

\section{El currículum en red según Efland}

Efland apuesta por un diseño del currículum que refleje a la vez la estructura de las disciplinas o campos del conocimiento y el modo de organizarse el conocimiento en la mente humana. Sostiene que un currículum de tales características fomenta la flexibilidad cognitiva, es decir la capacidad mental de observar elementos en común entre diferentes ideas o circunstancias y, por ende, de transferir conocimiento de 
unas situaciones a otras, como puede ser de una situación de aprendizaje a una situación fuera del aula. Si ponemos en práctica un diseño curricular integrado, al corresponderse la organización de la materia con el modo en que se almacenan y estructuran los conocimientos en la mente del individuo, se vuelve más fluido y aumenta exponencialmente el aprendizaje. Así pues, el currículum en red tiene, además de una razón de ser académica, una base psicológico-cognitiva.

A continuación reproduzco unos esquemas de Efland en los que se muestra, a un nivel muy básico y aclaratorio, cómo la retícula surge de la yuxtaposición de dos o más esquemas arboriformes (Efland, 1997). Los ejemplos 1 y 2 muestran dos agrupaciones posibles de una serie de objetos que presentan características en común; el ejemplo 3, la retícula que surge al combinar ambas agrupaciones.

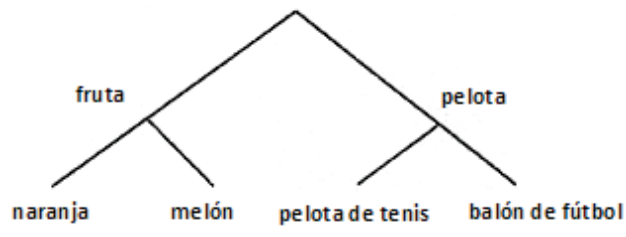

Fig. 1. Ejemplo 1

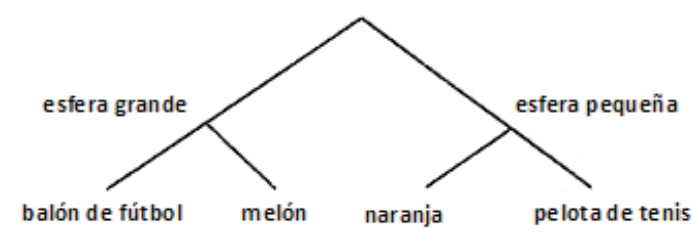

Fig. 2. Ejemplo 2

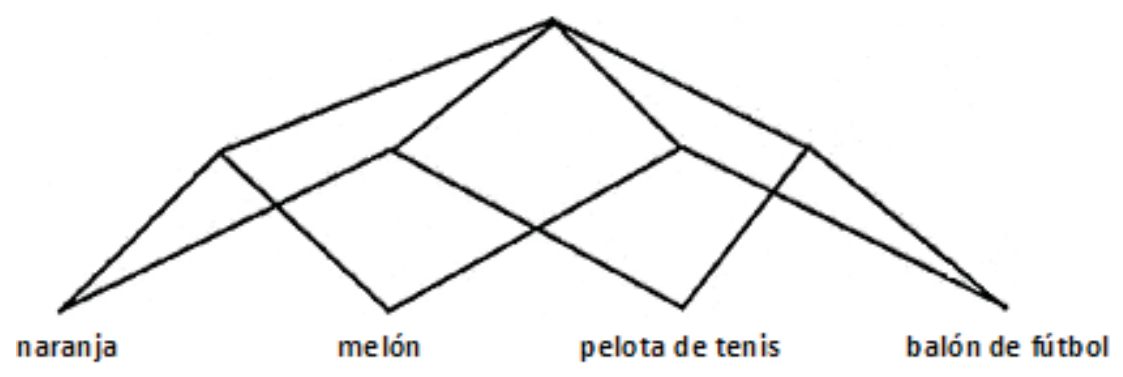

Fig. 3. Ejemplo 3

El siguiente ejemplo es una retícula en abstracto que pone todos sus componentes en relación. En este modelo me he basado para confeccionar la retícula que propongo más adelante para entrelazar las diferentes modalidades de la práctica musical.

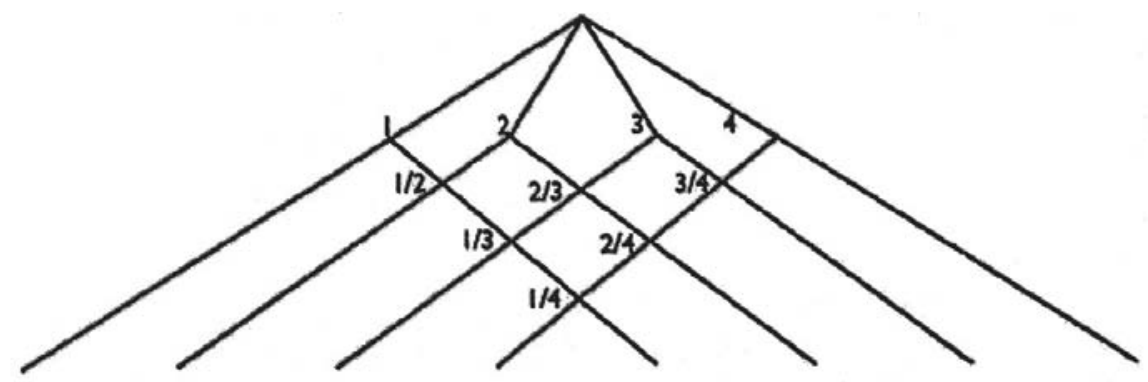

Fig. 4. Ejemplo 4 
En el marco de la enseñanza de las artes y las humanidades, la conveniencia de estructurar los contenidos en forma de retícula y no de árbol es especialmente manifiesta. La razón es que en estos campos las relaciones entre elementos deben producirse no tanto vertical como transversalmente, fomentando la transferencia de conocimiento y el análisis comparativo. Comparto la visión de Efland de que intentar estructurar en esquemas arboriformes los campos de las artes y las humanidades con el fin de simplificar los contenidos y los procesos de evaluación, no conduce sino a una desvirtuación de la materia y a la formación de conceptos ingenuos y aislados, que pueden incluso ser la base de futuros problemas de compresión (Efland, 1997). En los campos del saber en los que no hay verdades absolutas ni generalizaciones que valgan, es preferible abordar la materia a través del estudio de casos -Art is best taught through case studies (Eisner, 1987), y poner en relación el conocimiento recopilado a partir de esos casos mediante la aplicación de la retícula.

Efland recomienda individuar determinadas obras de arte como nodos que sirvan ya sea de punto de partida para el estudio de diversos temas, o de punto de llegada en el que convergen temas tratados con anterioridad:

\section{[...] la relación entre la obra de arte y la cultura es recíproca. Es decir, la obra de arte adquiere sentido cuando se ve en el contexto de la cultura, y la cultura se hace comprensible cuando se lee a través de su arte. Por esta razón, yo sugiero que los lugares en que la integración del conocimiento se maximiza dependen de las obras de arte como claves para la comprensión. (Efland, 2004)}

En su artículo Tintín, Bart Simpson y los Fanhunters. ¿De qué hablarían entre ellos?, Laura Trafí observa que esos nodos pueden consistir también en productos mediáticos de actualidad que nos ayuden a conectar con los intereses de los jóvenes y a captar con ello su atención. (Trafí, 1999).

\section{Por qué estructurar en red los contenidos de la materia de Música}

Naturalmente, las razones por las que Efland defiende la aplicación del currículum integrado en la materia de Arte, son igualmente válidas para la asignatura de Música. Si asumimos que los contenidos de la materia no deben simplificarse si no queremos desvirtuar su esencia, tenemos que buscar una manera de organizarlos que no sobrecargue al alumno. Pero en cualquier caso, la forma de impartir la materia debe evidenciar que en la producción musical convergen la influencia de prácticas musicales anteriores y la búsqueda de nuevas soluciones o formas de expresión, y que la práctica musical refleja una diversidad de factores culturales, históricos, sociales, políticos, económicos, etc. que confluyen en ella. Un currículum en red que pone de manifiesto las relaciones entre la música y otras disciplinas, y entre unas obras o fenómenos musicales y otros, respeta y refleja la naturaleza de la música. Recordemos la frase de Efland: Puesto que las obras de arte reflejan su 
entorno social, tienen un potencial incorporado para conectar con otros campos del conocimiento. En consecuencia, la asignatura de Música, como la de Educación plástica y visual, se presta idóneamente a que sus contenidos se comuniquen en forma de red.

A todo esto se suman las contribuciones del currículum musical integrado al desarrollo de las competencias básicas, una de las principales innovaciones del diseño curricular de la LOE respecto a los currículos anteriores, que enlaza directamente con la idea de flexibilidad cognitiva. Al constituirse las competencias básicas como el eje alrededor del cual se organiza el currículum, el saber conceptual pierde protagonismo en una idea de educación orientada hacia la práctica, hacia la capacidad de aplicar el saber más allá del aula. (Zaragozá, 2009).

Las diferentes facetas de la música que se ponen en práctica atendiendo a los bloques de contenidos ("Escucha", "Interpretación", "Creación" y "Contextos musicales" en $2^{\circ}$ y $3^{\circ}$ de la E.S.O., y "Audición y referentes musicales", "La práctica musical" y "Música y tecnologías" en $4^{\circ}$ ) implican de alguna manera el desarrollo de esas competencias. La competencia social y ciudadana, por ejemplo, se trabaja mediante la ejecución musical en grupo y el respeto de las pautas que presupone la interpretación conjunta; la competencia matemática, a partir del estudio de la teoría y el lenguaje musical y de la realización de ejercicios rítmicos. Se sobreentiende que la asignatura de Música apela a la competencia cultural y artística dado que se trata de una materia artística por definición. La competencia en comunicación lingüística se impulsa a través de los discursos construidos en el bloque 3 ("Contextos musicales"), que enlaza también con el tratamiento de la información y la competencia digital; etc. etc. Pero yo considero que todas estas competencias se pueden desarrollar ulteriormente si nos valemos del currículum en red tal y como propongo en el apartado siguiente, esto es, requiriendo la participación activa del alumnado en el tejido de las redes. A continuación resumiré el modo en que el currículum integrado impulsa cada una de las ocho competencias básicas establecidas por decreto:

- Competencia cultural y artística: un estudio de la música que pone en relación las diferentes manifestaciones musicales entre sí, así como esas manifestaciones y el contexto histórico y sociocultural que las envuelve, proporcionará al alumno las herramientas necesarias para el análisis y la comprensión de producciones o contextos musicales que desconoce, desde una postura abierta e indagadora a la vez que informada y crítica.

- Competencia en comunicación lingüística: no sólo se hará uso del lenguaje hablado para exponer contenidos en el marco del estudio teórico de la música, sino que los alumnos tendrán que aprender a verbalizar las relaciones que observan entre los componentes de la red y a explicitar la lógica de sus razonamientos. En lo que se refiere al comentario de audiciones, para describir aquello que se 
experimenta de forma sensorial hemos de recurrir a la metáfora, que no es sino la comparación de elementos de naturaleza diferente. La materia de Música, como la de Educación plástica y visual, contribuye también al desarrollo de la competencia en comunicación lingüística en la medida en que, como nos dice Eisner, escribir requiere la habilidad de ver, oír y percibir el mundo de manera que el escritor tenga un contenido que expresar y el deseo de compartirlo con otros. (Eisner, 1987) No me extenderé más en este punto porque no es el asunto que nos concierne principalmente en este trabajo, pero considero que el desarrollo de la competencia lingüística a través de la asignatura de Música puede ser el tema para un trabajo muy interesante y de gran envergadura.

- Competencia en el conocimiento y la interacción con el mundo físico: la percepción, por parte del alumno, de la realidad sonora que lo circunda se agudiza a través del desarrollo consciente de su sentido de la audición. Escuchar de forma consciente equivale a establecer conexiones. Cuantas más conexiones establezcamos en el presente, más conexiones podremos establecer en situaciones futuras.

- Tratamiento de la información y competencia digital: un currículum de estas características, que requiere el acercamiento a temas de diferente índole, exige por extensión el manejo de diversas fuentes de información (fuentes primarias y secundarias, grabaciones sonoras, partituras, textos musicológicos y de otras disciplinas, obras de referencia, datos estadísticos, etc.), así como distintos modos de procesamiento de esa información (lectura o escucha detenida, análisis, extracción de ideas, resumen, paráfrasis, asociación de conceptos...).

- Competencia social y ciudadana: como veremos en el siguiente apartado, esta competencia se puede poner en práctica, además de en la interpretación musical en grupo, a través del esfuerzo conjunto de la clase por elaborar una red de conocimientos; del fomento de una actitud abierta y respetuosa; y de la crítica constructiva ante las aportaciones de los compañeros. Si conseguimos que los alumnos se escuchen mutuamente, las ideas de unos inspirarán las de otros y la retícula se hará cada vez más grande.

- Competencia para aprender a aprender: esperar que sean los alumnos quienes tejan las redes entre los contenidos del retículo, aunque la guía del profesor suponga una ayuda importante, significa esperar de ellos que aprendan a reflexionar, a relacionar conceptos y a construir significado por sí mismos. En la medida en que tomen conciencia de la adquisición de esta capacidad, además de aprender a aprender también aprenderán que pueden aprender.

- Autonomía e iniciativa personal: la autonomía sólo podrá potenciarse en los niveles más básicos de construcción de la red, para los que sean suficientes los conocimientos que el alumno ha asimilado con anterioridad. También podrá 
estimularse con la asignación de tareas muy concretas que estén enteramente dentro de las posibilidades de cada alumno. Para establecer conexiones más complejas o individuar nuevos elementos que puedan incorporarse a la red por poseer características en común con otros componentes, probablemente sea imprescindible la ayuda del profesor.

La iniciativa personal se puede impulsar alentando a los alumnos a que participen y mediante el refuerzo positivo por parte del profesor. Si los alumnos perciben el producto de su trabajo y advierten que el profesor reconoce sus esfuerzos, aumentará su motivación intrínseca, la confianza en sí mismos y, por ende, su iniciativa.

- Competencia matemática: ésta es la única competencia que no soy capaz de vincular fácilmente a los efectos que produce la integración de los contenidos de la materia de Música, aunque si nos obstinamos, tal vez podríamos hablar del desarrollo de un «pensamiento geométrico» a partir de las formas trazadas en la retícula.

\section{Cómo aplicar el método de la creación guiada de redes de conocimiento}

La capacidad de asociación o flexibilidad cognitiva se desarrolla concretamente a través del ejercicio de identificar aspectos en común entre distintos conceptos o fenómenos, de modo que se amplíen progresivamente los componentes y los puntos de conexión de la red. Sostengo que el profesor no sólo debe pensar en estos términos a la hora de preparar las clases y hacer luego explícitas las conexiones ante los alumnos, dándoles ya hecha la tarea de relacionar, sino que conviene que aliente a los estudiantes a que sean ellos mismos quienes construyan la red. En el papel de guía, el profesor puede estimular la implicación del los alumnos a través de la formulación de preguntas que despierten su interés y curiosidad, y fomentar el aprendizaje significativo incitándoles a razonar con los conocimientos que ya poseían para construir, a partir de éstos, otros nuevos. Las redes de conocimiento creadas de manera conjunta se plasmarán visualmente en la pizarra y los alumnos deberán incluirlas asimismo en sus apuntes.

Al contar con la guía del profesor, pero insisto, sólo con la guía, los alumnos pueden progresar en el aprendizaje dentro de lo que Vygotsky denomina zona de desarrollo próximo. Ésta es la distancia entre el nivel real de desarrollo del alumno, determinada por su capacidad de resolver independiente un problema o una tarea, y su nivel de desarrollo potencial. Dicha distancia se salva con la ayuda de un profesor en calidad de guía o mediador. La ventaja de trabajar dentro de la zona de desarrollo próximo es que ésta afianza los conocimientos que ya posee el alumno al tiempo que lo estimula al máximo de sus posibilidades, pero sin rebasarlas. Al afianzarse sus conocimientos, el alumno se siente seguro y capaz. El reto de 
superar las dificultades que progresivamente se sitúan a su alcance, por otra parte, lo mantiene motivado. En consecuencia, considerar y respetar la zona de desarrollo próximo a lo largo de la creación guiada de redes de conocimiento, garantiza el avance en el proceso de aprendizaje.

Así pues, para que funcione con éxito el método de impartición de la asignatura que propongo, es de fundamental importancia que el profesor ayude a los alumnos a reflexionar, a confiar en sus capacidades y a aventurarse a comunicar sus pensamientos al resto de la clase. Sólo así podrá construirse un saber que sea producto del esfuerzo de todos. El interés del aprendizaje cooperativo radica en que el éxito y la satisfacción compartidos son mayores que los individuales, y en que la conjunción de esfuerzos, además de promover la cohesión social, suele conllevar una asimilación más profunda de los conocimientos adquiridos.

\section{El currículum en red y la investigación en la acción}

El carácter experimental de la puesta en práctica de un currículum integrado nos permite ponerlo en relación con lo que se conoce en pedagogía como investigación en la acción. La investigación en la acción es la observación crítica in situ de la propia práctica con el objetivo de mejorarla. A diferencia de lo que ocurre en la investigación convencional, son los mismos implicados en la realidad analizada quienes llevan a cabo la investigación en la acción. Ésta se estructura en un proceso cíclico de acción - observación - reflexión - nueva acción, al que subyace la idea de que la docencia es una actividad que siempre se puede optimizar y de que sus fines, los valores educativos, se persiguen sin que se lleguen a alcanzar nunca.

Al situarse a sí mismo en el punto de mira, el profesor investigador adquiere mayor conciencia de su modo de actuar, de aquello que daba por sentado, del grado en que las directrices institucionales limitan su actuación, de sus miedos e inseguridades, así como de sus aspiraciones más profundas. Hacer explícitas y criticables nuestras actuaciones «nos permite reorientar la práctica» en un proceso de continua búsqueda. (Contreras, 1994a).

Cuando propiciamos una situación de aprendizaje que implica a los alumnos en la configuración del transcurso de la clase, tenemos que estar muy atentos a que nuestro papel de guía surja el efecto deseado; a que los alumnos participen de forma activa y respetuosa; y a que el proceso sea fructífero y desemboque efectivamente en la producción de conocimiento. Como probablemente los alumnos no estarán habituados a semejante organización de las clases, conviene hacerlos conscientes de la idea de que están siendo partícipes de un aprendizaje experimental. Darles a conocer el interés de nuestros objetivos para que éstos se conviertan también en sus objetivos puede suscitar en los alumnos una actitud colaboradora. La experiencia educativa se convierte entonces en un experimento en el que todos participan activamente con un fin común. Como nos dice Contreras, 
Las actuaciones deben analizarse en su calidad educativa, esto es, en relación al valor educativo que tienen por sí mismas como experiencia

(Contreras, 1994a)

\section{Un ejemplo de red: la integración de las distintas prácticas musicales}

En el siguiente ejemplo de red trato de reflejar cómo pueden solaparse las diferentes formas de práctica musical -la composición, la interpretación, la improvisación y la audición-, el aprendizaje del lenguaje musical y el estudio de diversas manifestaciones musicales desde un punto de vista (etno) musicológico. Los colores de las dobles líneas que demarcan los hexágonos indican qué formas de práctica musical se solapan en cada caso.

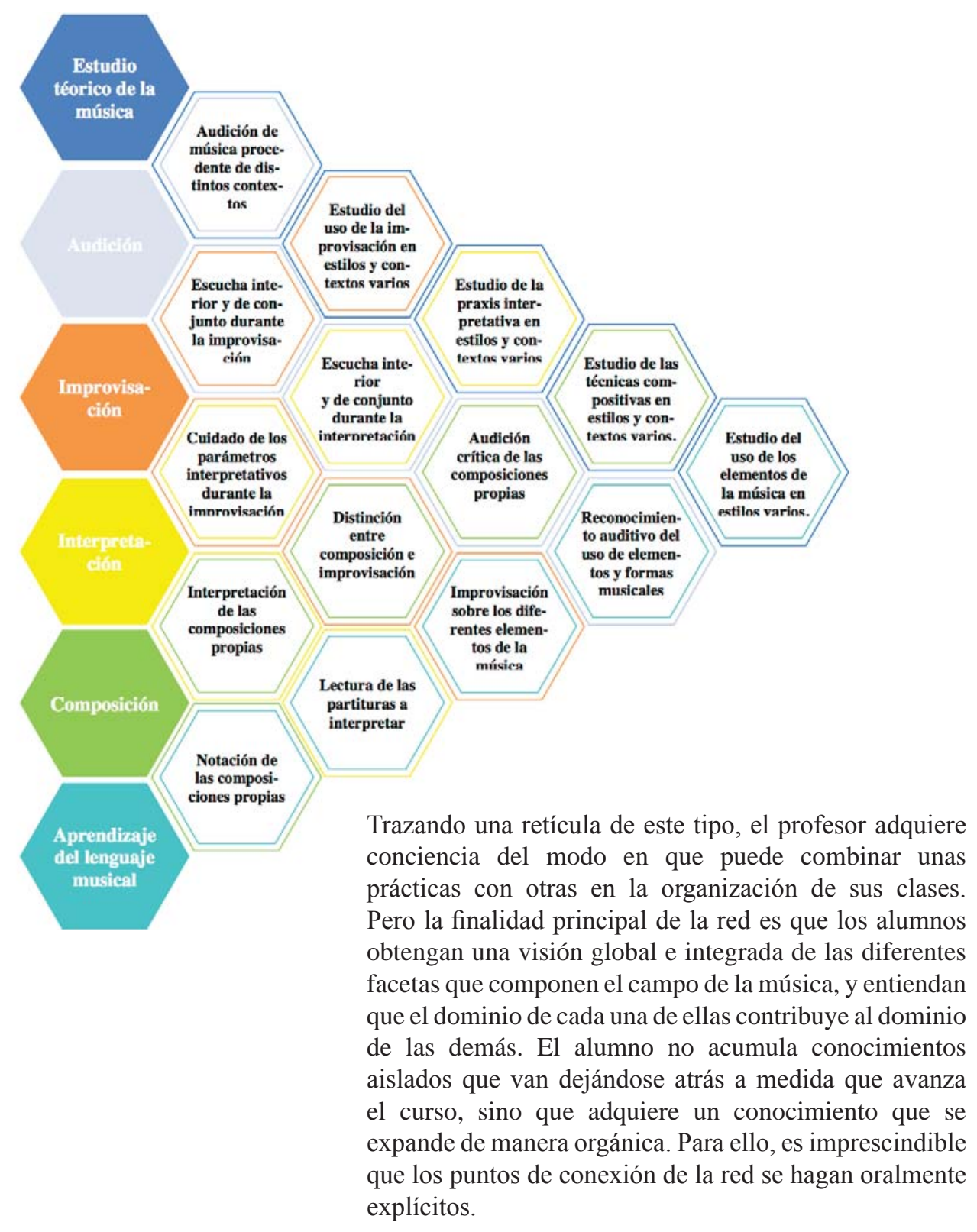




\section{Un ejemplo de nodo: Cantares}

La audición que propongo a continuación sirve de nodo para articular diversos contenidos y objetivos en forma de propuesta didáctica. Al volver periódicamente sobre el elemento que constituye el hilo conductor, los alumnos aprenden a focalizar la atención al tiempo que asocian diferentes temas y conceptos.

Por el contexto que envuelve la composición de Joan Manuel Serrat y los versos de Antonio Machado, esta propuesta didáctica nos permite establecer vínculos directos con la materia impartida en las asignaturas de Ciencias sociales y Castellano: lengua y literatura en $4^{\circ}$ de la E.S.O. Así pues, no sólo aglutina contenidos del currículo de Música, sino que integra a la vez varias disciplinas. Ello nos ayudará a movernos en la zona de desarrollo próximo y reforzará considerablemente el aprendizaje significativo. Podemos solicitar la colaboración del/de la profesor/a de Lengua y literatura, de manera que el análisis literario que enmarco en la segunda sesión de la propuesta metodológica se realice en su clase.

\section{Audición de Cantares}

Propuesta didáctica

Compositor e intérprete: Joan Manuel Serrat Letra: Antonio Machado (Campos de Castilla, Proverbios y cantares LIV, I y XXIX) y Joan Manuel Serrat

Curso : $4^{\circ}$ E.S.O.

Tiempo de impartición: 8 sesiones de 55 min.

\section{Contenidos del currículum:}

\section{Bloque 1. Audición y referentes musicales:}

- «Audición, reconocimiento, análisis y comparación de músicas de diferentes géneros y estilos $[\ldots] \gg$.

- «Interés, respeto y curiosidad por la diversidad de propuestas musicales, así como por los gustos musicales de otras personas».

\section{Bloque 2. La práctica musical:}

- «Ámbitos profesionales de la música. Identificación y descripción de las distintas facetas y especialidades en el trabajo de los músicos». (figura del cantautor).

- «Interpretación de piezas vocales e instrumentales aprendidas de oído [...]».

- «Utilización de diversas técnicas, recursos y procedimientos compositivos en la improvisación, la elaboración de arreglos y la creación de piezas musicales».

\section{Bloque 3. Música y tecnologías:}

- «El papel de las tecnologías en la música. Transformación de valores, bitos, consumo y gusto musical como consecuencia de los avances tecnológicos de las últimas décadas». 


\section{Competencias básicas:}

Competencia lingüística - el poema de Machado atestigua el valor del lenguaje como vehículo para la expresión de ideas y sentimientos. Los alumnos también tendrán que hacer uso del lenguaje para comunicar sus opiniones al resto de la clase y contribuir a la creación de redes de conocimiento.

Competencia social y ciudadana - el contenido del texto permite tratar temas como la democracia y la justicia social. La dinámica interactiva de clase implica el respeto del turno de palabra y de las opiniones ajenas. La realización de la tarea final por grupos prevé asimismo la cooperación.

Competencia cultural y artística - la audición guiada de música permite conocer, comprender, apreciar y valorar críticamente diferentes manifestaciones culturales y artísticas, utilizarlas como fuente de enriquecimiento y disfrute y considerarlas como parte del patrimonio de los pueblos. La interpretación y la composición impulsarán las habilidades técnicas de los alumnos y les proporcionarán una experiencia de la práctica musical en primera persona.

\section{Objetivos:}

- Conocer la figura del cantautor y algunos aspectos relevantes del género.

- Entender que la música y la poesía, como el resto de manifestaciones artísticas, han sido un arma poderosa tanto de opresión como de oposición en muchos conflictos políticos.

- Reconocer los instrumentos musicales que intervienen y las relaciones que se establecen entre la voz y el acompañamiento instrumental.

- Percibir los cambios de ritmo y carácter y los distintos usos de la voz, e inferir los efectos que persigue el cantante con todo ello.

- Emitir juicios de valor fundamentados, referidos tanto a la composición como a la interpretación.

- Trabajar la entonación, la utilización de la voz y algunos recursos interpretativos.

- Promover la cohesión del grupo a través de la interpretación conjunta. La carga emotiva del texto debería contribuir a ello.

- Fomentar la capacidad creativa, la expresión lingüística y la destreza rítmica en la composición de un rap. 


\section{Propuesta metodológica:}

\begin{tabular}{|c|c|c|}
\hline Sesión & Contenidos y actividades & Metodología \\
\hline \multirow{3}{*}{$1^{\mathrm{a}}$} & Audición inicial de Cantares & $\begin{array}{l}\text { Audición desprovista } \\
\text { explicaciones previas }\end{array}$ \\
\hline & $\begin{array}{l}\text { Familiarización con los términos } \\
\text { "cantautor" y "canción de } \\
\text { protesta". Conocer algunos } \\
\text { aspectos relevantes del género, } \\
\text { como son laimplicación a menudo } \\
\text { socio-política y el carácter } \\
\text { reivindicativo; su proliferación } \\
\text { en las décadas de } 1960 \text { y 1970, } \\
\text { ligada a los movimientos de } \\
\text { protesta; y el lenguaje musical } \\
\text { generalmente sencillo, destinado } \\
\text { a preservar la comprensibilidad y } \\
\text { la supremacía del mensaje. }\end{array}$ & $\begin{array}{l}\text { Creación guiada de redes de } \\
\text { conocimiento a través de preguntas } \\
\text { del tipo “¿Qué creéis que puede ser } \\
\text { un cantautor?" o ¿Por qué creéis } \\
\text { que se caracteriza la canción de } \\
\text { protesta? } \\
\text { Audición focalizada. En una } \\
\text { segunda audición los alumnos } \\
\text { deberán concentrarse en averiguar } \\
\text { si predomina la letra o la música y } \\
\text { preguntarse por qué. }\end{array}$ \\
\hline & $\begin{array}{l}\text { Contextualización histórica y } \\
\text { literaria de los Proverbios y } \\
\text { cantares de Machado y de la } \\
\text { canción de Serrat (alusión a la } \\
\text { Generación del 98, al franquismo } \\
\text { y al exilio). }\end{array}$ & $\begin{array}{l}\text { Creación guiada de redes de } \\
\text { conocimiento. Los alumnos se } \\
\text { remitirán a los conocimientos } \\
\text { adquiridos paralelamente en las } \\
\text { asignaturas de Ciencias Sociales } \\
\text { y Castellano: lengua y literatura } \\
\text { para elaborar conjuntamente la } \\
\text { contextualización de Cantares. }\end{array}$ \\
\hline $2^{\mathrm{a}}$ & $\begin{array}{l}\text { Estudio del contenido, los } \\
\text { recursos literarios y la rima de los } \\
\text { versos de Machado y Serrat. Se } \\
\text { examinará asimismo la manera en } \\
\text { que Serrat combina sus propios } \\
\text { versos con los de Machado. }\end{array}$ & $\begin{array}{l}\text { Análisis escrito individual del texto } \\
\text { atendiendo a preguntas concretas. } \\
\text { Puesta en común de los análisis } \\
\text { individuales. }\end{array}$ \\
\hline \multirow[t]{2}{*}{$3^{\mathrm{a}}$} & $\begin{array}{l}\text { Análisis formal de Cantares a } \\
\text { partir de la relación melodía- } \\
\text { estrofa; reconocimiento de los } \\
\text { instrumentos que intervienen, } \\
\text { descripción delarelaciónentrevoz } \\
\text { y acompañamiento instrumental, } \\
\text { percepción de los cambios de } \\
\text { ritmo y de carácter, apreciación } \\
\text { de los distintos usos de la voz. }\end{array}$ & $\begin{array}{l}\text { Análisis auditivo guiado de } \\
\text { la canción. Se responderá } \\
\text { individualmente y por escrito a } \\
\text { preguntas concretas a partir de la } \\
\text { audición repetida sin partitura pero } \\
\text { con la ayuda de la letra impresa. } \\
\text { Puesta en común. }\end{array}$ \\
\hline & $\begin{array}{l}\text { Comentario y valoración estética } \\
\text { de la interpretación }\end{array}$ & $\begin{array}{l}\text { Expresión y argumentación de } \\
\text { opiniones personales }\end{array}$ \\
\hline
\end{tabular}




\begin{tabular}{|c|c|c|}
\hline $4^{\mathrm{a}}$ & $\begin{array}{l}\text { Interpretación conjunta a una } \\
\text { voz de la canción, con la posible } \\
\text { asignación de algunos solos, a } \\
\text { capella y con acompañamiento } \\
\text { instrumental en directo o de } \\
\text { karaoke a través de youtube. }\end{array}$ & $\begin{array}{l}\text { Disponiendo de la letra impresa } \\
\text { o proyectada, se cantará } \\
\text { conjuntamente atendiendo a } \\
\text { los parámetros interpretativos } \\
\text { aprendidos y a la escucha interior } \\
\text { y de conjunto. Se memorizará } \\
\text { parte de la letra, por ejemplo los } \\
\text { versos de Machado, para fomentar } \\
\text { su valoración y su interiorización, } \\
\text { ejercitar la memoria y focalizar la } \\
\text { atención en la calidad interpretativa. }\end{array}$ \\
\hline \multirow[t]{2}{*}{$5^{\mathrm{a}}$} & $\begin{array}{l}\text { El caso Rasteryaev: otro ejemplo } \\
\text { de cantautor. Comparación entre } \\
\text { el videoclip oficial de la canción } \\
\text { Kombaynery y el video casero } \\
\text { original, cuya rápida difusión en } \\
\text { youtube elevó a la fama a Igor } \\
\text { Rasteryaev. Esta comparación } \\
\text { nos permitirá enlazar con los } \\
\text { temas de la comercialización de } \\
\text { la música y la globalización. }\end{array}$ & $\begin{array}{l}\text { Visualizaciónyanálisis comparativo } \\
\text { oral de un vídeo casero y un } \\
\text { videoclip oficial. Creación guiada } \\
\text { de redes de conocimiento a partir de } \\
\text { las observaciones de los alumnos. }\end{array}$ \\
\hline & $\begin{array}{l}\text { Presentación de una tarea para } \\
\text { casa: búsqueda de una canción de } \\
\text { protesta que se presentará en } 2-3 \\
\text { minutos al resto de la clase. }\end{array}$ & Indicaciones del profesor. \\
\hline $6^{\mathrm{a}}$ & $\begin{array}{l}\text { Audición comentada de } \\
\text { canciones de otros cantautores } \\
\text { que propongan los alumnos y } \\
\text { comparación de éstas con los } \\
\text { ejemplos anteriores. }\end{array}$ & $\begin{array}{l}\text { Exposiciones breves de los alumnos, } \\
\text { audiciones y comentario conjunto. }\end{array}$ \\
\hline \multirow[t]{2}{*}{$7^{\mathrm{a}}$} & $\begin{array}{l}\text { El rap como estilo en el que impera } \\
\text { igualmente la letra, portadora de } \\
\text { mensajes sociales o políticos. A } \\
\text { partir de la audición de algunos } \\
\text { ejemplos se observará que el } \\
\text { ritmo, que viene dado por la letra, } \\
\text { se constituye como el elemento } \\
\text { musical principal del rap. }\end{array}$ & $\begin{array}{l}\text { Audición y creación guiada de redes } \\
\text { de conocimiento. }\end{array}$ \\
\hline & $\begin{array}{l}\text { Presentación de una tareafinal para } \\
\text { casa, que consistirá en componer } \\
\text { un rap con texto reivindicativo en } \\
\text { grupos de dos o tres alumnos. Se } \\
\text { propondrá entre todos una serie } \\
\text { de pautas que sirvan como guía } \\
\text { para la realización de la tarea. }\end{array}$ & $\begin{array}{l}\text { Indicaciones del profesor } \mathrm{y} \\
\text { aportaciones de los alumnos. }\end{array}$ \\
\hline
\end{tabular}




\begin{tabular}{|l|l|l|}
\hline $8^{\mathrm{a}}$ & $\begin{array}{l}\text { Interpretación por grupos de los } \\
\text { raps de composición propia. }\end{array}$ & $\begin{array}{l}\text { Interpretación, escucha y crítica } \\
\text { constructiva. }\end{array}$ \\
\hline
\end{tabular}

\section{Criterios de evaluación:}

- Actitud abierta y tolerante hacia las audiciones propuestas

- Participación activa en la creación de redes de conocimiento

- Razonamiento de los juicios emitidos y respeto de las opiniones de los demás

- Relación de conceptos

- Percepción auditiva

- Puesta en práctica de las técnicas interpretativas aprendidas y respeto de las pautas básicas de la interpretación en grupo

- Creatividad en la composición

- Aplicación del conocimiento adquirido en las tareas a realizar

Otros temas colindantes que pueden abordarse en ulteriores sesiones a partir de esta misma audición son:

- Las relaciones interartísticas - la creación de obras artísticas basada en otras obras de la misma disciplina o de disciplinas diferentes. (música y pintura; pintura y literatura; música y literatura; literatura y cine; cine y música; música y danza; etc.).

- Las relaciones entre texto y música en la música vocal. Observación de soluciones varias en distintas épocas y estilos.

- El arte al servicio de la política (por ejemplo, el uso de la música como instrumento de propaganda política por parte del régimen nacionalsocialista).

\section{Conclusiones}

Para que la experiencia del alumno en el horario lectivo pueda servirle más allá del aula, podemos concluir que el mapa de conceptos al que se enfrente tendrá que ser, en palabreas de Efland, una representación adecuada del panorama social 
y cultural en el que vivimos. (Efland, 1997) Para ello, deberá parecerse más al retículo. El currículum ha de ayudar al alumno a relacionar lo que aprende en el contexto académico con su experiencia personal. La medida en que se alcance este objetivo será la medida en que el aprendizaje cobre sentido para el alumno y, por extensión, la medida en que se mantendrá motivado. Parsons, igualmente partidario del currículum integrado, explica así su esencia en relación con el concepto del «mundo de la vida» impulsado por Edmund Husserl y Jürgen Habermas:

La integración se produce cuando los estudiantes confieren sentido por sí mismos a sus experiencias varias de aprendizaje, cuando las aúnan para formar una visión de su mundo y del lugar que ocupan en él. [...]. Los defensores del currículum integrado se preocupan fundamentalmente por hacer que aprender sea significativo para los alumnos. (Parsons, 2004)

Enlazando con la idea de Piaget de que el desarrollo cognitivo es un proceso activo, el currículum en red presupone que es el alumno quien construye el significado de lo que se aprende. Percatarse de que es él quien genera conocimiento, en lugar de que éste le sea inculcado de forma unidireccional por la figura externa del profesor, puede resultarle muy gratificante. Y si el aprendizaje se construye conjuntamente entre los estudiantes, no sólo será gratificante la sensación de contribuir a los resultados, sino que además se potenciará la conciencia social del alumnado.

Supongo que una de las razones por las que no se ha difundido ampliamente la práctica del currículum en red es que sería difícil ponerse de acuerdo sobre la manera de evaluar sus resultados. La mejora en la capacidad del alumno de aplicar los conocimientos adquiridos a distintas situaciones de la vida no es tan fácil de evaluar como los "pequeños pasos" de un aprendizaje sistemático, ni tampoco lo son el resto de objetivos que persigue implícitamente el currículum integrado, como son el aumento de la implicación del alumno, el desarrollo de su capacidad de expresión y diálogo, y un largo etcétera. Pero opino que es más importante que los alumnos aprendan, que el hecho de que el sistema pueda demostrar con datos numéricos su eficacia.

En una sociedad en la que los cambios se producen a una velocidad vertiginosa y los estímulos y las fuentes de información son cada vez más numerosos y diversificados, el sistema educativo ha de abogar porque los estudiantes estén en condiciones de percibir esa información con criterio y discernimiento. Se vuelve especialmente patente la necesidad de implementar métodos educacionales innovadores como el del currículum en red, cuya meta principal es que los alumnos aprendan a reflexionar y a valerse por sí mismos.

Sostengo que un currículum de estas características vela y aboga por la libertad del individuo, que emana de la habilidad de pensamiento, del sentido crítico y de la capacidad de imaginar realidades alternativas. 


\section{Referencias bibliográficas}

Arnaus, R. y Contreras, J. (1993). Una experiencia de la investigación en la acción. Cuadernos de Pedagogía, no 220, 85-90.

Contreras, J. (1994a). La investigación en la acción: ¿Qué es? Cuadernos de Pedagogía, $\mathrm{n}^{\mathrm{o}} 224,8-12$.

Contreras, J. (1994b). La investigación en la acción: ¿Cómo se hace? Cuadernos de Pedagogía, n² 224, 14-19.

Efland, A. D. (1997). El currículum en red: una alternativa para organizar los contenidos de aprendizaje. Kikiriki, n ${ }^{0}$ 42/43, 96-109.

Efland, A. D. (2004). Arte y cognición. La integración de las artes visuales en el currículum. Barcelona: Octaedro.

Efland, A. D., Freedman, K. y Stuhr, P. (2003). La educación en el arte posmoderno. Barcelona: Paidós Ibérica.

Eisner, E. W. (1987). Procesos cognitivos y currículum. Una base para decidir lo que hay que enseñar. Barcelona: Martínez Roca.

Parsons, M. (2004). Art and Integrated Curriculum. En E. W. Eisner y M. D. Day (eds.). Handbook of Research and Policy in Art Education. Nueva Jersey: Lawrence Erlbaum Associates.

Trafí, L. (1999). Tintín, Bart Simpson y los Fanhunters. ¿De qué hablarían entre ellos? Cuadernos de Pedagogía, no 285, 55-60.

Zaragozà, J. Ll. (2009). Didáctica de la Música en la educación secundaria. Competencias docentes y aprendizaje. Barcelona: Graó. 